\title{
All-digital AFSK modem with Viterbi detection for TT\&C CubeSat transceiver
}

\author{
Rubem Vasconcelos Pacelli, Antonio Macilio Pereira de Lucena, João Cesar Moura Mota
}

\begin{abstract}
Audio-Frequency Shift Keying (AFSK) with continuous phase modulation and non-coherent demodulation is widely used on the telecommand channel of the most TT\&C transceivers for CubeSat. However, this solution has low efficiency in terms of power. In this paper, a new all-digital AFSK modem is proposed. The demodulator uses the Viterbi algorithm to perform the maximum likelihood sequence detection (MLSD) and gives an expressive gain when compared to non-coherent detection. Original mathematical analyzes are presented to explain the proposed system. Some performance results, determined via computational simulation, are presented and discussed.
\end{abstract}

Keywords-AFSK, CubeSat, coherent demodulation, Viterbi algorithm.

\section{INTRODUCTION}

The CubeSat standard made possible to launch several miniaturized satellites in the past two decade for different applications in science and engineering [1]. The rapid growth of launches has prompted studies of new spectrally efficient modulation techniques to mitigate the overcrowding bandwidth allocated for satellite communication [2]. However, it can be seen that one of the most used modulation schemes on telecommand channel for CubeSat TT\&C (Telemetry, Tracking and Command) transceiver is still the classical AFSK (Audio-Frequency Shifting Keying) modulation [3], [4]. Its choice is mainly due to the advantage of being used in conjunction with the amateur radio protocol AX.25. This solution allows amateur radio operators around the world to collect information from the satellites and, in some cases, improve the system's communication ability [5], [6]. Furthermore, AFSK modem can be easily implemented on inexpensive programmable devices, facilitating its utilization [7].

The AFSK modulation reported in literature is implemented as a CPFSK (Continuous-Phase Frequency Shifting Keying) signal [8], [9]. This signaling has an advantage over the nonmemory FSK scheme because the phase continuity decreases the spectral side-lobe, making the modulation spectrally more efficient. Another attractive factor is its constant envelope that permits the utilization of non-linear amplifier without spectrum regrowth [10].

For applications in CubeSat TT\&C transceiver, the AFSK demodulator is commonly implemented as a non-coherent detector [8], [9]. The main reason for that is the simplicity,

Rubem Vasconcelos Pacelli, Grupo de Pesquisa em Processamento de Sinais e da Informação (GPSI), Universidade Federal do Ceará (UFC), Fortaleza-CE, e-mail: rubem.engenharia@gmail.com; Antonio Macilio Pereira de Lucena, Instituto Nacional de Pesquisas Espaciais (INPE) and Universidade de Fortaleza (UNIFOR), Fortaleza-CE, e-mail: antonio.lucena@inpe.br; João Cesar Moura Mota, Grupo de Pesquisa em Telecomunicações em Fio (GTEL) and GPSI, Fortaleza-CE, e-mail: mota@gtel.ufc.br. This work was partially funded by CAPES/FUNCAP program (88887.363460/2019-00) and CNPq. considering the AFSK demodulator is on-board of the satellite where there is a strong limitation of resources. This choice has a direct impact in the speed of data transmission for the telecommand link. For the most product available in the market, the speed is limited to $1200 \mathrm{bps}$.

In this paper, a new all-digital coherent AFSK modem is presented. The proposed demodulator performs the optimum detection of the received signal using the Viterbi algorithm. Such a strategy improves the performance of the system in terms of bit error rate (BER). This gain can have some impact in the design of antennas (on-board or ground) or in the power of the earth station transmitter.

It is worthy to emphasize that the AFSK demodulator shall be embedded in reprogrammable logical device, such as FPGA (Field-Programmable Gate array), giving much more flexibility to the the TT\&C module. The bit rate of the link or even all the architecture of demodulator can be modified by software even after the satellite launching.

The main contributions of this article are:

1) Presentation of new completely digital architectures for the AFSK modulator with continuous phase modulation.

2) Description and analysis of the new architecture, also completely digital, for the optimum detection of the AFSK signal with continuous phase via Viterbi detection.

3) Derivation of an upper-bound of the error probability for the new all-digital AFSK demodulator.

4) Discussion of the results of computer simulation to evaluate and compare the performance of the proposed systems with the non-coherent demodulation.

This paper is organized in the following way: In section II, the architecture of modulator and the signal model is introduced. In section III, the demodulator for coherent Viterbi detection is derived. In section IV, an upper-bound for the error probability is derived. In sections V and VI, the results and conclusions are exposed, respectively.

\section{AFSK MODULATOR}

The AFSK modulation is a Binary Frequency Shifting Keying (BFSK) scheme that uses tones $f_{0}$ and $f_{1}$ to represent the bits " 1 " and " 0 ", respectively, where $\Delta f=f_{1}-f_{0}=1$ $\mathrm{kHz}$. When used a bit rate of $1200 \mathrm{bit} / \mathrm{s}$, this scheme is referred as AFSK1200 [8]. The BFSK signal can be implemented either by switching the $f_{0}$ and $f_{1}$ frequencies or by changing the phase of the carrier. In the last case, the signal generated belongs to CPFSK class because its phase does not have discontinuities.

Let us define $\boldsymbol{I}=\left[I_{0}, I_{1}, \ldots\right]$ as a vector of symbols independent and identically distributed, where $I_{k} \in\{-1,1\}$ 
is the $k$ th symbol obtained from the mapping of a stream of bits and $k \in \mathbb{N}$. This sequence passes through a upsampling expander block that introduces $N-1$ zeros among the symbols [11]. Therefore, after this point, the duration of each symbol corresponds to $N$ samples. The output signal is

$$
I_{e}[n]=\sum_{k=0}^{\infty} I_{k} \delta[n-k N],
$$

where $\delta[n]$ is the Kronecker delta function. The time-varying phase of the carrier is defined as

$$
\Phi[n, \boldsymbol{I}]=4 \pi T f_{d}\left(I_{e}[n] * q[n]\right),
$$

where $T$ is the bit period, $f_{d}=\Delta f / 2$ is the peak frequency deviation, $q[n]$ is the phase pulse, given by

$$
q[n]=\left\{\begin{aligned}
0 & \text { if } n<0, \\
\frac{n+1}{2 N} & \text { if } 0 \leq n \leq N-1, \\
\frac{1}{2} & \text { if } n>N-1 .
\end{aligned}\right.
$$

and $*$ denotes the operation of convolution.

Let us analyze $\Phi[n, \boldsymbol{I}]$ for the interval $i N \leq n \leq$ $(i+1) N-1$, that corresponds to the duration of one symbol. Substituting Eq. (1) in Eq. (2), we have

$$
\begin{aligned}
\Phi[n, \boldsymbol{I}] & =4 \pi T f_{d} \sum_{k=0}^{i} I_{k} q[n-k N] \\
& =2 \pi h \sum_{k=0}^{i-1} I_{k} q[n-k N]+2 \pi h I_{i} q[n-i N] \\
& =\pi h \sum_{k=0}^{i-1} I_{k}+2 \pi h I_{i} q[n-i N] \\
& =\theta_{i}+\theta\left[n, I_{i}\right],
\end{aligned}
$$

where $h=2 T f_{d}$ is the modulation index,

$$
\theta_{i}=\pi h \sum_{k=0}^{i-1} I_{k}
$$

is the phase memory until the instant $i N-1$ and $\theta\left[n, I_{i}\right]=$ $2 \pi h I_{i} q[n-i N]$ is the linear phase transition during the interval from $i N$ up to $(i+1) N-1$.

The modulated carrier signal, $s[n, I]$, can be generated in the quadrature form, that is,

$$
\begin{aligned}
s[n, \boldsymbol{I}]= & \sqrt{\frac{2 E}{T}} \cos (\Phi[n, \boldsymbol{I}]) \cos \left(2 \pi f_{c} n T_{s}\right) \\
& -\sqrt{\frac{2 E}{T}} \sin (\Phi[n, \boldsymbol{I}]) \sin \left(2 \pi f_{c} n T_{s}\right) \\
= & I[n, \boldsymbol{I}] \cos \left(2 \pi f_{c} n T_{s}\right)-Q[n, \boldsymbol{I}] \sin \left(2 \pi f_{c} n T_{s}\right),
\end{aligned}
$$

where $I[n, \boldsymbol{I}]$ and $Q[n, \boldsymbol{I}]$ are the phase and quadrature components of $s[n, I]$, respectively, $E$ is the bit energy, $f_{c}$ is the carrier frequency and $T_{s}=T / N=1 / f_{s}$ is sampling period, being $f_{s}$ the sampling frequency.

The Fig. 1 shows a block diagram that summarizes the process of modulation. The symbol $\bullet$ denotes the input signal of the block.

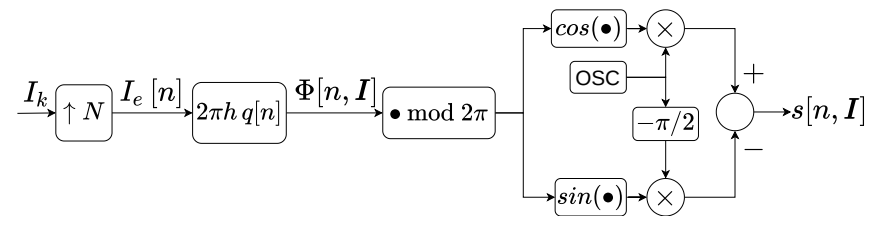

Fig. 1. Block diagram of modulator.

In this figure, $x \bmod 2 \pi$ indicates the remainder of the division of $x$ by $2 \pi$. This operation converts $\Phi[n, \boldsymbol{I}]$ to the interval of phase between 0 and $2 \pi$, avoiding we have numeric overflow in the processing. The block denominated "OSC" indicates a local oscillator that produces a discrete-time cosine centred at $f_{c}$. The last step of the modulator, a D/A (Digitalto-Analog) converter is used to obtain the analog signal, $s(t, I)$, which is passed to RF (Radio Frequency) stage to be transmitted.

For CPM modulation, all possible values of $\theta_{i}$ can be described by a finite set $\phi$. For an index modulation $h=m / p$, where $m$ and $p$ are integers, prime to each other and $p$ being even, the set $\phi$ is given by [12]

$$
\boldsymbol{\phi}=\left\{0, \frac{\pi m}{p}, \frac{2 \pi m}{p}, \ldots, \frac{(2 p-1) \pi m}{p}\right\} .
$$

For 1200AFSK, the symbol period is $T=1 / 1200=$ $833.33 \mu \mathrm{s}$, the peak frequency deviation is $f_{d}=\Delta f / 2=500$ $\mathrm{Hz}$ and the modulation index is $h=2 T f_{d}=5 / 6$. Therefore, the terminal phase states for AFSK signal is

$$
\begin{aligned}
\boldsymbol{\phi} & =\left\{\phi_{0}, \phi_{1}, \ldots, \phi_{11}\right\} \\
& =\left\{0, \frac{5 \pi}{6}, \frac{10 \pi}{6}, \ldots, \frac{55 \pi}{6}\right\} .
\end{aligned}
$$

Note that, by Eq. (8), there are 12 possible values of phase distributed in the interval from 0 up to $10 \pi$. The Fig. 2 shows the phase tree of AFSK signal which the initial phase is $\theta_{0}=$ $\phi_{0}=0$.

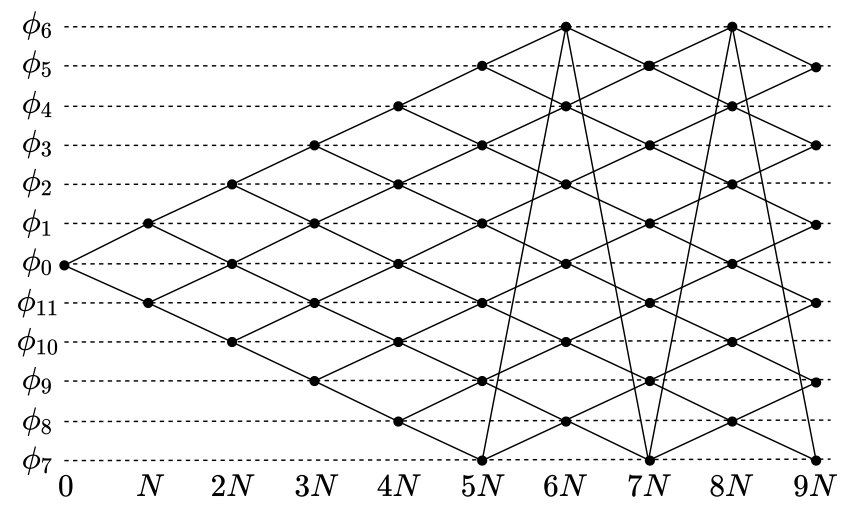

Fig. 2. Phase tree of AFSK signal

The phase of AFSK signal is added to $5 \pi / 6$ or to $-5 \pi / 6$ depending on the transmitted symbol be +1 or -1 , respectively. In the case when the resulting phase is out of limits defined by $\phi_{0}$ and $\phi_{11}$, the phase is wrapped to remain inside the limits. The behaviour of phase tree depicted in Fig. 2 is our start point to development of the Viterbi detection explained hereafter. 


\section{AFSK DEMODULATOR}

The channel is considered AWGN (Additive White Gaussian Noise). After to pass through the RF front-end of the receiver, the signal is delivered to demodulator, that performs the A/D (Analog-to-Digital) conversion. The obtained signal is

$$
r[n]=s[n, \boldsymbol{I}]+w[n],
$$

where $s[n, \boldsymbol{I}]$ is given by Eq. (6) and $w[n]$ is AWGN (Additive White Gaussian Noise) with zero mean and spectral power density equal to $N_{o} / 2 T_{s}$. Before the sampling, the spectral power density of the correspondent AWGN analog noise is $N_{o} / 2$.

We assume that demodulator has synchronization circuits that recover the symbol time, carrier phase and frequency.

Let us express $w[n]$ in the band-pass form

$w[n]=w_{I}[n] \cos \left(2 \pi f_{c} n T_{s}\right)-w_{Q}[n] \sin \left(2 \pi f_{c} n T_{s}\right)$,

where $w_{I}[n]$ and $w_{Q}[n]$ are the baseband quadrature components of the stochastic process $w[n]$ [13]. Substituting Eq. (6) and Eq. (10) into Eq. (9), we have

$$
\begin{aligned}
r[n]= & \left(I[n, \boldsymbol{I}]+w_{I}[n]\right) \cos \left(2 \pi f_{c} n\right) \\
& -\left(Q[n, \boldsymbol{I}]+w_{Q}[n]\right) \sin \left(2 \pi f_{c} n\right) \\
= & \hat{I}[n] \cos \left(2 \pi f_{c} n\right)-\hat{Q}[n] \sin \left(2 \pi f_{c} n\right) .
\end{aligned}
$$

When the signal depends on its past states, a sequence of symbols must be considered for detection in order to explore the memory inherent in the modulation process. Let us define a finite vector of transmitted symbols, $\boldsymbol{I}_{i}=\left[I_{0}, I_{1}, \ldots, I_{i-1}\right]$, the optimum receiver shall find a path through the phase tree that maximizes the likelihood function, given by $p_{\boldsymbol{v}}\left(\boldsymbol{V} \mid \boldsymbol{I}_{i}\right)$, the joint conditional probability density function of $\boldsymbol{v}=$ $\left[v_{0}, v_{1}, \ldots, v_{i-1}\right]$, where $v_{k}$ is the $k$ th output of the correlator and $\boldsymbol{V}$ is the observation of $\boldsymbol{v}$ [12].

Thus the optimum receiver is a correlator followed by a maximum likelihood sequence detector (MLSD) that search for the sequence through the phase tree (described in Fig. 2) whose correlation is maximum. The correlation computation is used as metric for the search by the MLSD. However, this structure is not feasible in practice because the number of possibles paths grows exponentially for large values of $i$. The Viterbi algorithm is a efficient method that performs this search recursively for each state. Let us define the correlation metric for $\boldsymbol{I}_{i}$ during the interval $0 \leq n \leq N i-1$ as

$$
J\left(\boldsymbol{I}_{i}\right)=\sum_{n=0}^{N i-1} r[n] s\left[n, \boldsymbol{I}_{i}\right] .
$$

The metric for the new sequence $\boldsymbol{I}_{i+1}=\left[\boldsymbol{I}_{i}, I_{i}\right]$ can be calculated as [14], [15]

$$
J\left(\boldsymbol{I}_{i+1}\right)=J\left(\boldsymbol{I}_{i}\right)+v\left(I_{i}, \theta_{i}\right),
$$

where $v_{i} \triangleq v\left(I_{i}, \theta_{i}\right)$ is the transition metric for the initial state $\theta_{i} \in \phi$ and the received symbol $I_{i}$ during the interval $i N \leq n \leq(i+1) N-1$. This value is given by

$$
\begin{aligned}
v\left(I_{i}, \theta_{i}\right)= & \sum_{n=0}^{N-1} r[n+N i] \\
& \cdot \cos \left(2 \pi f_{c} n T_{s}+\theta\left(n, I_{i}\right)+\theta_{i}\right) .
\end{aligned}
$$

Substituting Eq. (11) into Eq. (14) and ignoring the second harmonics components, we have

$$
\begin{aligned}
v\left(I_{i}, \theta_{i}\right)= & \sum_{n=0}^{N-1} \hat{I}[n+N i] \cos \left(\theta\left[n, I_{n}\right]+\theta_{i}\right) \\
& +\sum_{n=0}^{N-1} \hat{Q}[n+N i] \sin \left(\theta\left[n, I_{n}\right]+\theta_{i}\right) .
\end{aligned}
$$

The Fig. 3 shows a block diagram for computation of this transition metric used by the Viterbi algorithm (VA). In this diagram, "LPF" indicates a low-pass filter that removes the second harmonic.

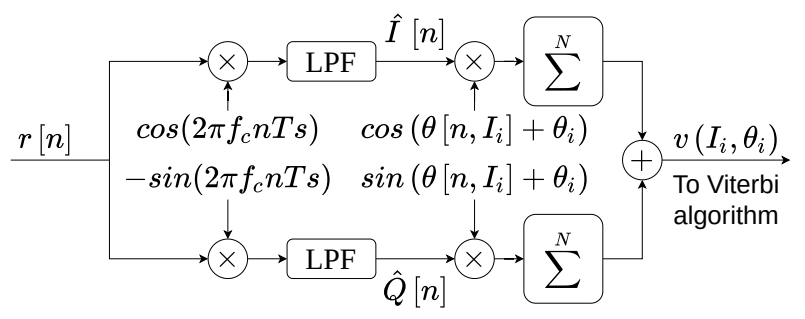

Fig. 3. Coherent Viterbi demodulator.

For a binary transition, there are two metric for each state. The Viterbi algorithm compares these two metrics and discards the path having the lower metric. The another path with the greater metric is saved and is called the survivor [12]. However, the Fig. 2 reveals that just half of all state can be reached in a give instant $i N$. In $(i+1) N$, just the another half can be reached. This behavior continues alternately. Therefore, for a efficient computation, just half of states must be taken in consideration.

Note that, in the instant $i N$, each state has its own survivors, $\boldsymbol{I}_{i}$, and its own metric, $J\left(\boldsymbol{I}_{i}\right)$. An important issue about this algorithm is the convergence of all survivors to an unique vector. If we have advanced this process, it can be noted that from the fifth most recent symbol onwards it has a high probability of being identical [12].

\section{UPPER-BOUND FOR BIT ERROR PROBABILITY}

In this section, an upper-bound for the bit error probability is derived for the proposed digital AFSK demodulator. For continuous-time CPFSK, there is a large amount of paper which the theoretical bit error probability is analyzed. These articles have reported that is not possible to derive an exact mathematical expression for the bit error probability. [16], [17]. However, one can derive an upper-bound that gives a tight approximation for the actual BER. The bit error probability can be determined asymptotically by the term corresponding to the minimum Euclidean distance, $d_{m i n}$, between two signals associated with different symbol vectors, $\boldsymbol{I}^{(r)}$ and $\boldsymbol{I}^{(s)}$, over a given time interval $i T$ [16], [18]. Pelchat has shown that there is a maximum value that $d_{\min }$ can reach for a time arbitrarily large [19]. This limit allows a derivation of an upper-bound for the bit error probability in the continuous-time CPFSK system.

We derive a bit error probability upper-bound for the present discrete-time CPFSK signal using the same approach. Suppose 
that we have two signals, $s\left[n, \boldsymbol{I}^{(0)}\right]$ and $s\left[n, \boldsymbol{I}^{(1)}\right]$, corresponding to two sequences, $\boldsymbol{I}^{(0)}$ and $\boldsymbol{I}^{(1)}$. These sequences must be different in their first symbol. The squared Euclidean distance between this two discrete signals over the interval $0 \leq n \leq i N-1$ is

$$
\begin{aligned}
\Delta_{0,1}^{2}(i)= & \sum_{n=0}^{i N-1}\left(s\left[n, \boldsymbol{I}^{(0)}\right]-s\left[n, \boldsymbol{I}^{(1)}\right]\right)^{2} \\
= & \sum_{n=0}^{i N-1} s^{2}\left[n, \boldsymbol{I}^{(0)}\right]+\sum_{n=0}^{i N-1} s^{2}\left[n, \boldsymbol{I}^{(1)}\right] \\
& -2 \sum_{n=0}^{i N-1} s\left[n, \boldsymbol{I}^{(0)}\right] s\left[n, \boldsymbol{I}^{(1)}\right] .
\end{aligned}
$$

The first and the second term in the right side of Eq. (16) can be rewritten as

$$
\begin{aligned}
& \frac{2 E}{T} \sum_{n=0}^{i N-1} \cos ^{2}\left(2 \pi f_{c} n T_{s}+\Phi\left[n, \boldsymbol{I}^{(l)}\right]\right)= \\
& \frac{E i N}{T}+\frac{E}{T} \sum_{n=0}^{i N-1} \cos \left(4 \pi f_{c} n T_{s}+2 \Phi\left[n, \boldsymbol{I}^{(l)}\right]\right),
\end{aligned}
$$

where $l=0$ or 1 . Suppose that $f_{s}$ is an integer multiple of $f_{c}$. Hence, $N_{c}=f_{s} / f_{c}$ is the number of samples per carrier cycle. For $N \gg N_{c}$, the value of phase $\Phi\left[n, \boldsymbol{I}^{(l)}\right]$ is approximately constant for each carrier period and the result of the summation on the right side of Eq. (17) is practically zero. Substituting Eq. (17) into Eq. (16) and recalling that $T=N T_{s}$, we have

$$
\begin{aligned}
\Delta_{0,1}^{2}(i) & =\frac{2 E i}{T_{s}}-2 \frac{2 E}{N T_{s}} \sum_{n=0}^{i N-1} \cos \left(2 \pi f_{c} n T_{s}+\Phi\left[n, \boldsymbol{I}^{(0)}\right]\right) \\
& \cdot \cos \left(2 \pi f_{c} n T_{s}+\Phi\left[n, \boldsymbol{I}^{(1)}\right]\right) \\
& =\frac{2 E i}{T_{s}}-\frac{2 E}{N T_{s}} \sum_{n=0}^{i N-1} \cos \left(\Phi\left[n, \boldsymbol{I}^{(1)}\right]-\Phi\left[n, \boldsymbol{I}^{(0)}\right]\right) \\
& =\frac{2 E}{T_{s}}\left(i-\frac{1}{N} \sum_{n=0}^{i N-1} \cos (\Phi[n, \boldsymbol{\xi}])\right) \\
& =\frac{2 E}{T_{s}} \delta_{0,1}^{2}(i),
\end{aligned}
$$

where $\boldsymbol{\xi}=\boldsymbol{I}^{(1)}-\boldsymbol{I}^{(0)}$ and

$$
\delta_{0,1}^{2}(i)=i-\frac{1}{N} \sum_{n=0}^{i N-1} \cos (\Phi[n, \boldsymbol{\xi}])
$$

is the squared Euclidean distance normalized by the bit energy of the discrete baseband signal, that is $2 E / T_{s}$.

For this modulation scheme, the difference sequence $\xi_{o}=$ $\{ \pm 2, \mp 2,0,0, \ldots\}$ provides the maximum value for the minimum normalized squared Euclidean distance, $\delta_{\min }^{2}$ [18]. The upper-bound of the minimum normalized squared Euclidean distance, $\delta_{B}^{2}(h)$, can be calculated evaluating $\delta_{0,1}(i)^{2}$ for $\xi=\xi_{o}$, that is:

$$
\begin{aligned}
\delta_{B}^{2}(h)=2-\frac{1}{N} & \sum_{i=0}^{2 N-1} \cos \left(\Phi\left[n, \boldsymbol{\xi}_{o}\right]\right) \\
=2-\frac{1}{N} & \left(\sum_{i=0}^{N-1} \cos \left(\frac{2 \pi h n}{N}\right)\right. \\
& \left.\quad+\sum_{n=0}^{N-1} \cos \left(2 \pi h-\frac{2 \pi h n}{N}\right)\right) .
\end{aligned}
$$

The equation Eq. (20) is numerically calculated for $h=$ $5 / 6$ and the result is $\delta_{B}^{2}(h)=2.33$. For $N>4$, the value of $\delta_{B}^{2}(h)$ always converge to 2.33. Since this inequation is always attended for practical purpose, we have the same value of $\delta_{B}^{2}(h)$ for any feasible value of $N$.

Note that the upper-bound of the minimum normalized squared Euclidean distance for the equivalent continuous-time system, given by [12], [16]

$$
d_{B}^{2}(h)=2\left(1-\frac{\sin 2 \pi h}{2 \pi h}\right),
$$

has the same value found by Eq. (20) when $h=5 / 6$.

Finally, following the same arguments found in [12] and [19] for the case of the continuous and binary CPFSK, we have determined the upper-bound of the bit error probability for the present discrete architecture that is given by

$$
P_{e} \leq K Q\left(\sqrt{\delta_{\min }^{2} \frac{E}{N_{o}}}\right),
$$

where $K=2$ is the number of paths having the minimum distance. For an observation interval greater than 2 symbols time, $\delta_{\min }^{2}=\delta_{B}^{2}(h)$.

\section{NUMERICAL RESULTS}

In this section, we present some performance results of the proposed AFSK modem, which were obtained from computer simulation. The power spectrum of the transmitted AFSK signal and the bit error rate of the system are determined.

The model has the following parameters:

- Bit period, $T=833.33 \mu \mathrm{s}$;

- Carrier frequency, $f_{c}=120 \mathrm{kHz}$;

- Sampling frequency, $f_{s}=1 / T_{s}=480 \mathrm{kHz}$;

- Samples per symbol, $N=T / T_{s}=400$;

- Index modulation, $h=5 / 6$;

- Energy per bit to noise power density, $E / N_{o}=$ From 0 $\mathrm{dB}$ up to $11 \mathrm{~dB}$;

In order to evaluate the performance of the proposed modulator, the Fig. 4 shows the spectrum of the present all-digital AFSK modulator, generated by computer simulation, along with the theoretical power spectral density of the AFSK signal, which was determined by Eq. (3.4-61) from [12]. It is possible to notice that the power density of the proposed all-digital AFSK modulator has both good symmetry around $f_{c}$ and perfectly matches with the theoretical power spectral density. Moreover, is important to note that such symmetry is hard to be accomplish in analog modulator. 


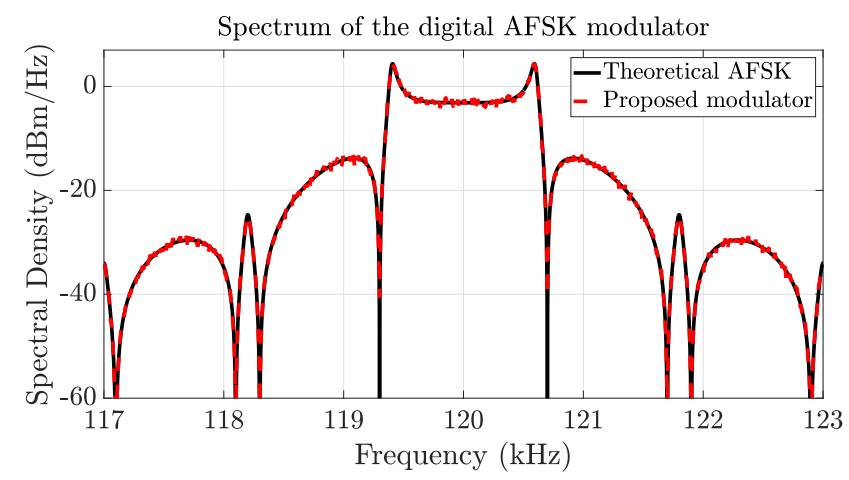

Fig. 4. Spectrum of digital AFSK modulator.

The Fig. 5 shows the BER of the coherent AFSK demodulator and its upper-bound, given by Eq. (22), for different values of $E / N_{o}$. It is possible to note that the upper-bound of the bit error probability provides a tight approximation for the estimated BER. We also plot the theoretical bit error probability for the non-coherent AFSK demodulation that is the usual technique used in most of CubeSat TT\&C transceivers available on the market. This curve was determined by Eq. (4.5-54) from [12]. For a BER equals to $10^{-5}$, the Viterbi demodulator achieves an improvement of $5 \mathrm{~dB}$, when compared with the non-coherent technique.

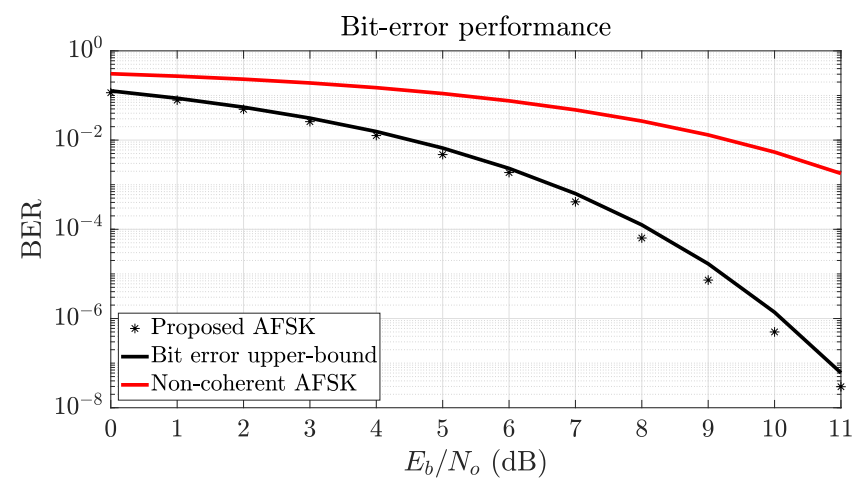

Fig. 5. Bit-error performance.

\section{CONCLUSiOns}

In this paper, we presented an all-digital AFSK modem for application in CubeSat TT\&C transceiver. The architectures of the modulator and demodulator as well as its mathematical analysis for description and evaluation of the conceived system were presented.

For the detection of the symbols, a new maximum likelihood detector, based on the Viterbi detection and operating under the discrete signal, was developed and analyzed. The upperbound for the bit error probability of this new architecture was determined and it was found that its value is equal to the upper-bound of the equivalent continuous CPFSK [16].

As the results, the spectrum of the AFSK modulator exhibited a perfect matching with the theoretical shape of the equivalent CPFSK signal. The upper-bound derived in this paper provides a tight approximation for the bit error performance of the simulated system. When compared to the non-coherent demodulation, the Viterbi detection achieved a improvement of $5 \mathrm{~dB}$, approximately, at a BER of $10^{-5}$.

As a future perspective for this work, one can cite the design of the symbol and carrier synchronization modules for the AFSK demodulator.

\section{ACKNOWLEDGEMENTS}

Thanks are due to CNPq (Conselho Nacional de Desenvolvimento Científico e Tecnológico) for found partially this research.

\section{REFERENCES}

[1] M. Emanuellia, L. Bettiola, M. Grulicha, J.E.G. Gonzaleza, J. Atchisona, L.L. Péreza and J. Sotudeha. Nanosatellites and their demand for changes in space policy. 67th International Astronautical Congress (IAC), September 2016.

[2] J. Šate, R. Trops, E. Briede, A. Orbidāns, M. Donerblics and R. Pabērzs. Concept of spectrally efficient communication subsystem. Space Research Review, Vol.4, 2016.

[3] B. Klofas. CubeSat Communications System Table. https://www.klofas.com/comm-table/. Accessed on January 2020.

[4] Satnogs statistics. https://db.satnogs.org/stats/. Accessed on January 2020.

[5] M.R. Maheshwarappa and C.P. Bridges. Software defined radios for small satellites. NASA/ESA Conference on Adaptive Hardware and Systems (AHS). pp. 172-179. July 2014.

[6] P. Muri and J. McNair. A survey of communication sub-systems for intersatellite linked systems and CubeSat missions. Journal of Communications. pp.290-308. April 2012.

[7] L.S. Velasco and A.R. Aranda. Software Based AFSK Generation on Arduino. XIII Technologies Applied to Electronics Teaching Conference (TAEE). pp. 1-5. June 2018.

[8] D. J. M. Peralta. Uma abordagem em rádio definido por software para operações em terra de pequenos satélites. Instituto Nacional de Pesquisas Espaciais (INPE), 2018.

[9] A. Addaim, A. Kherras and E.B. Zantou. Design of Low-cost Telecommunications CubeSat-class Spacecraft. Aerospace Technologies Advancements, p.293, 2010.

[10] G.T. Zhou. Analysis of spectral regrowth of weakly nonlinear power amplifiers. In 2000 IEEE International Conference on Acoustics, Speech, and Signal Processing.Vol. 5. pp. 2737-2740. June 2000.

[11] A.V. Oppenheim. Discrete-time signal processing. Pearson Education India. 1999.

[12] J.G. Proakis and M. Salehi. Digital communications. New York: McGraw-hill. 2007.

[13] A.L. Garcia. Probability and random processes for electrical engineering. Pearson Education India. 1994.

[14] B. Sklar. How I learned to love the trellis. IEEE Signal Processing Magazine. pp.87-102. 2003.

[15] M. Zourob. Detection, Receivers, and Performance of CPFSK and CPCK. 2013.

[16] T. Aulin and C.E. Sundberg. CPM - An efficient constant amplitude modulation scheme. International Journal of Satellite Communications. pp.161-186. 1984.

[17] Schonhoff, T. Symbol error probabilities for M-ary CPFSK: Coherent and noncoherent detection. IEEE Transactions on Communications, 24(6), pp.644-652. 1976.

[18] T. Aulin and C. Sunderberg. Continuous phase modulation-Part I: full response signalling. COM-29. pp.196-209. 1981.

[19] M.G. Pelchat, R.C. Davis and M.B. Luntz. Coherent demodulation of continuous phase binary FSK signals. International Foundation for Telemetering. 1971. 